\title{
The Condylar Cutoff Sign
}

\section{Quantifying Lateral Femoral Condylar Hypoplasia in a Complete Discoid Meniscus}

\author{
Chul-Won Ha MD, Yong Seuk Lee MD, \\ Jae Chul Park MD
}

Received: 22 February 2008/Accepted: 25 July 2008/Published online: 20 August 2008

(C) The Association of Bone and Joint Surgeons 2008

\begin{abstract}
We describe the condylar cutoff sign, a radiographic sign in knees with a discoid lateral meniscus, for diagnosis of complete discoid meniscus. We retrospectively reviewed tunnel-view radiographs of 100 patients (100) knees including 50 with complete discoid lateral meniscus and 50 with normal menisci. All patients were 18 years of age or older. All of the knees were arthroscopically examined. We developed a method to measure the prominence of the femoral condyle adjacent to the intercondylar notch on a tunnel view of the knee. The prominence ratio, the ratio of the medial and lateral condylar prominence, was compared and analyzed. The measurements were performed by three observers on two separate occasions to determine reliability. The intraobserver and interobserver variability study revealed high reliability with correlation coefficients ranging from 0.86 to 0.99 . Using a cutoff ratio of 0.8 , we observed a major difference between the two groups and found the ratio had $76 \%$ sensitivity, $96 \%$ specificity, $95 \%$ positive predictive value, and $80 \%$ negative predictive value in every set of
\end{abstract}

Each author certifies that he has no commercial associations. Each author certifies that his institution has approved or waived approval for the reporting of this case and that all investigations were conducted in conformity with ethical principles of research.

C.-W. Ha, Y. S. Lee, J. C. Park

Department of Orthopedic Surgery, Samsung Medical Center, Sungkyunkwan University School of Medicine, Seoul,

South Korea

\section{Y. S. Lee $(\bowtie)$}

Department of Orthopaedic Surgery, Korea University Ansan Hospital, 516 Gozan-dong, Danwon-gu, Ansan 425-707,

South Korea

e-mail: smcos1@hanmail.net measurements. The sign has high specificity and reliability but might not apply to younger patients.

Level of Evidence: Level II, diagnostic study. See the Guidelines for Authors for a complete description of levels of evidence.

\section{Introduction}

Discoid meniscus is a common anatomic variation in the knee with a reported incidence ranging from 5\% to $16.6 \%$ $[7,14]$. The clinical manifestations and treatment strategy of symptomatic discoid meniscus of a knee has been described [2, 3, 5, 9, 12]. The diagnosis of discoid meniscus historically has been made based on the patient's history, symptoms, and findings of physical examination, which include clicking, snapping, tightness, or a catching sensation in the knee. Magnetic resonance imaging, arthrography, and arthroscopy currently are the definitive diagnostic tools $[16,17]$, but come at a high cost, inconvenience, and risks associated with surgery.

Plain radiography has been considered an ancillary tool for the diagnosis of discoid meniscus. Although various indirect signs such as lateral joint space widening, hypoplasia of the lateral femoral condyle, hypoplasia of the lateral tibial spine, high fibular head, and cupping of the lateral tibial plateau have been reported, the diagnostic value of these signs is questionable $[8,14,16]$.

We identified a distinctive finding on plain radiography of the knee, which we named the condylar cutoff sign. The condylar cutoff sign refers to a decreased prominence of the lateral femoral condyle adjacent to the intercondylar notch so it appears the prominent part of the condyle has been cut off as compared with the normal appearance of the condyle (Fig. 1). Condylar cutoff is a better way of 


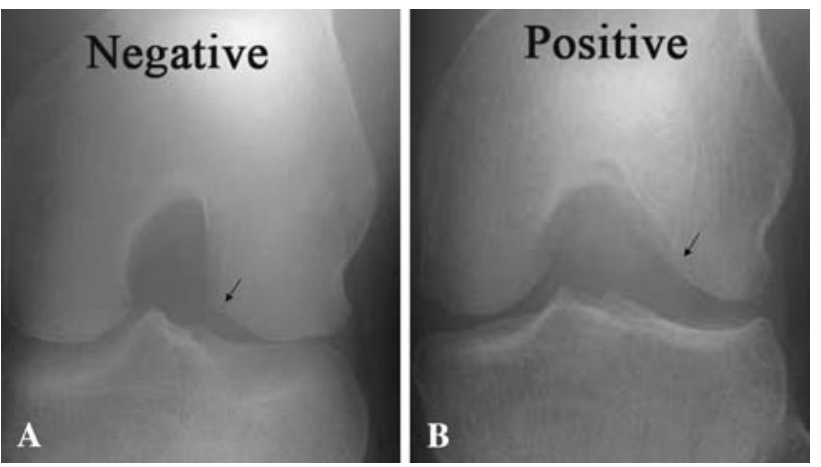

Fig. 1A-B Tunnel-view radiographs show (A) negative and (B) positive cutoff signs. (A) The arrow indicates the normal prominence of the lateral condyle adjacent to the intercondylar notch. Normal lateral meniscus was confirmed by arthroscopy in this knee. (B) The arrow indicates the decreased prominence of the lateral condyle adjacent to the intercondylar notch. Complete discoid lateral meniscus was confirmed by arthroscopy in this knee.

observing hypoplasia of the lateral femoral condyle. Our experience suggests the condylar cutoff sign is a simple and reliable radiographic sign for diagnosis of complete discoid meniscus in certain cases, such as for patients who have difficulty because of cost and access [15].

In this study we defined the condylar cutoff sign and (1) compared the prominence ratio between patients with lateral discoid meniscus and normal subjects, (2) determined the sensitivity, specificity, and positive and negative predictive values of the sign according to different cutoff points for diagnosis of complete discoid meniscus on simple radiography, and (3) determined the interobserver and intraobserver reliability of radiographic assessment of the sign.

\section{Materials and Methods}

We retrospectively reviewed 100 knees (100 patients) that were examined arthroscopically and showed no degenerative changes. Fifty knees had a complete discoid lateral meniscus and 50 were normal. We identified 50 consecutive patients with discoid lateral meniscus who had undergone arthroscopy for definitive diagnosis or treatment. Arthroscopy was performed if a patient had persistent locking, a block to extension, or severe pain with a loud click despite 3 months of nonoperative treatment. The 50 patients with normal knees were similarly recruited retrospectively: all had arthroscopy for medial meniscal or patellofemoral disorders. Complete discoid lateral meniscus was defined as the lateral meniscus fully covering the lateral tibial plateau with a round inner margin as seen on arthroscopic examination $[9,18]$. Patients with a history of trauma and younger than 18 years were excluded to avoid bias from immaturity. The two groups were comparable in demographic characteristics in terms of age (average, 34 years in both groups; range, 19-50 years in the discoid group, 23-56 years in the normal group) and gender distribution ( 22 males, 28 females in the discoid group; 29 males, 21 females in the normal group). Our Institutional Review Board granted an exemption for approval and patient consent.

All patients had standardized tunnel-view radiographs of the knee. We used the tunnel view because we thought it would provide the best image around the intercondylar notch area. The patients were placed prone with the knee flexed approximately $40^{\circ}$ with the foot supported by a foam block. The central beam was directed caudally toward the knee at a $40^{\circ}$ angle from the vertical plane (Fig. 2) [6].

We developed a method to measure the condylar prominence of the femoral condyles adjacent to the intercondylar notch to clearly define and quantify the condylar cutoff sign. To normalize any error from magnification of the film, we measured the condylar prominence of the medial and lateral femoral condyles adjacent to the intercondylar notch. Lines $\mathrm{A}\left(\mathrm{A}^{\prime}\right)$ were drawn through the outermost points of the femoral condyle medially and laterally. Line $B$ was drawn through the lowest points of the femoral condyles. Points a and $\mathrm{a}^{\prime}$ intersect lines $\mathrm{A}\left(\mathrm{A}^{\prime}\right)$ and line B. Point $\mathrm{c}$ is the highest point in the intercondylar notch. Lines $\mathrm{C}\left(\mathrm{C}^{\prime}\right)$ are the lines through points $\mathrm{c}$ and $\mathrm{a}\left(\mathrm{a}^{\prime}\right)$. The prominences $\mathrm{P}\left(\mathrm{P}^{\prime}\right)$ were measured as the longest distance between the prominences of the condyles and lines $\mathrm{C}\left(\mathrm{C}^{\prime}\right)$. The prominence ratio was defined as $\mathrm{P}^{\prime} / \mathrm{P}$ (Fig. 3).

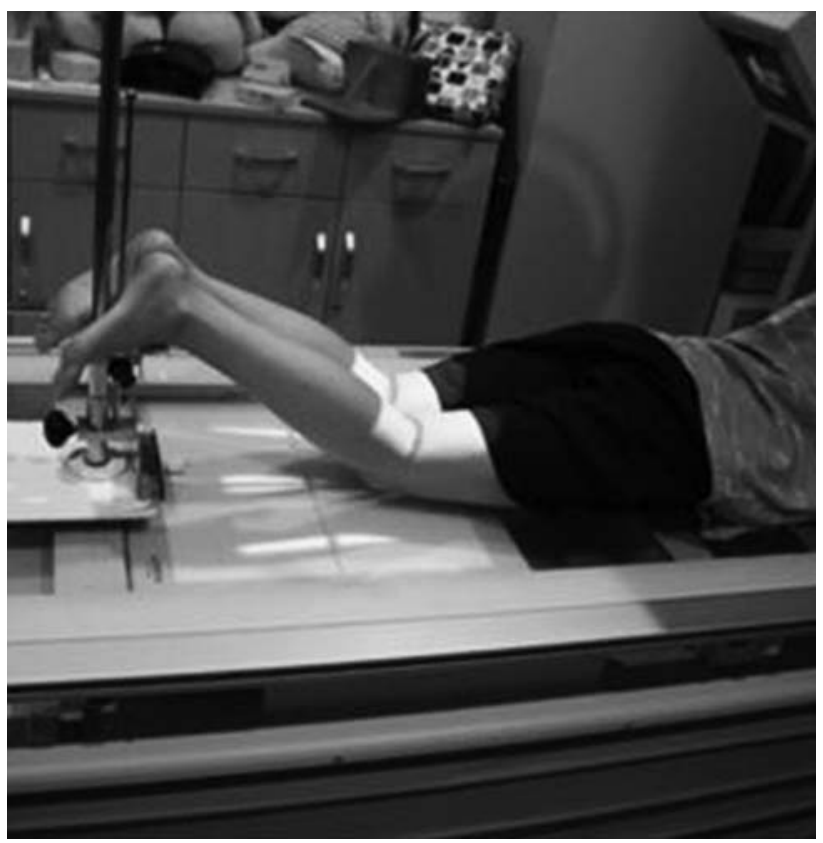

Fig. 2 The patient was prone with the knee flexed approximately $40^{\circ}$ and the foot supported by a sponge or supporter. The central beam was directed caudally toward the knee at a $40^{\circ}$-angle from the vertical plane. 


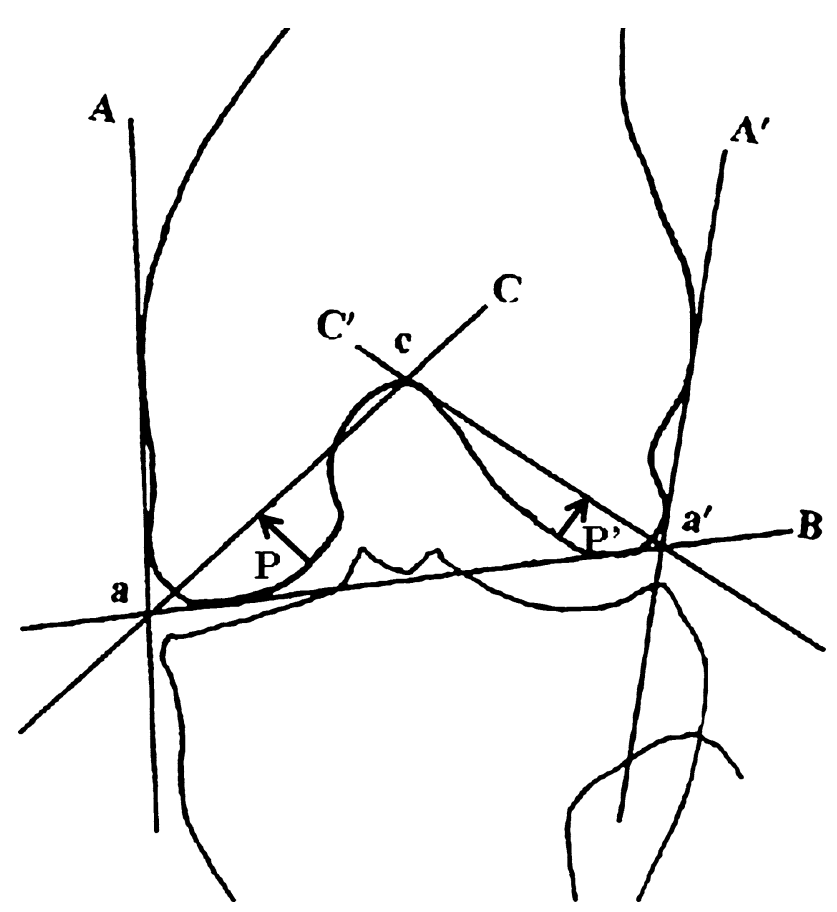

Fig. 3 A schematic drawing of the measurement to produce the prominence ratio for the radiographs in Fig. 1 is shown. Lines $A\left(A^{\prime}\right)$ were drawn through the outermost points of the femoral condyle medially and laterally. Line $B$ was drawn through the lowest points of the femoral condyles. Points a and $\mathrm{a}^{\prime}$ are the intersections of lines $\mathrm{A}\left(\mathrm{A}^{\prime}\right)$ and line B. Point $\mathrm{c}$ is the highest point in the intercondylar notch. Lines $\mathrm{C}\left(\mathrm{C}^{\prime}\right)$ are the lines through points $\mathrm{c}$ and $\mathrm{a}\left(\mathrm{a}^{\prime}\right)$. The prominences $\mathrm{P}\left(\mathrm{P}^{\prime}\right)$ were measured as the longest distance between the prominences of the condyles and lines $\mathrm{C}\left(\mathrm{C}^{\prime}\right)$. The prominence ratio was defined as $\mathrm{P}^{\prime} / \mathrm{P}$.

Three orthopaedic surgeons (PJC, SJI, AHK) blinded to the diagnosis performed the measurements. Interobserver and intraobserver reliability were analyzed by interclass and intraclass correlation coefficients. The measurements were repeated for the same radiographs at least 3 weeks after the first measurements for each observer, producing six sets of measurements for the 100 knees.

We compared the average values of the prominence ratio $\left(\mathrm{P}^{\prime} / \mathrm{P}\right)$ in the two groups using a two-tailed t-test. To produce a reliable cutoff point of the prominence ratio for diagnosing discoid lateral meniscus we chose different cutoff points $(0.5,0.6,0.7,0.8)$ and analyzed each by the chi square test. Numerous definitions were used to calculate the percentages [4]. The sensitivity, specificity, and positive and negative predictive values according to different cutoff points also were analyzed by calculating the percentages. We used SPSS program (SPSS 11.0, Chicago, IL) for all analyses.

\section{Results}

The discoid group had a lesser $(\mathrm{p}<0.0001)$ prominence ratio than normal knee group $(0.716 \pm 0.087$ in the discoid
Table 1. Prominence ratios

\begin{tabular}{lcl}
\hline Prominence & Discoid group & Normal group \\
\hline Medial condylar prominence $(\mathrm{P})$ & $12.67 \pm 1.97$ & $13.39 \pm 1.75$ \\
Lateral condylar prominence $\left(\mathrm{P}^{\prime}\right)$ & $9.07 \pm 1.80$ & $11.86 \pm 1.61$ \\
Prominence ratio $\left(\mathrm{P}^{\prime} / \mathrm{P}\right)$ & $0.716 \pm 0.087^{*}$ & $0.886 \pm 0.078^{*}$ \\
\hline
\end{tabular}

Values are mean \pm standard deviation; ${ }^{*} \mathrm{t}=-11.071 ; \mathrm{p}<0.0001$.

Table 2. Definitions of sensitivity, specificity, positive predictive value, and negative predictive value

\begin{tabular}{l} 
Sensitivity $=\frac{\text { true positive }}{\text { true positive }+ \text { false negative }} \times 100$ \\
Specificity $=\frac{\text { true negative }}{\text { true negative }+ \text { false positive }} \times 100$ \\
Positive predictive value $=\frac{\text { true positive }}{\text { true positive }+ \text { false positive }} \times 100$ \\
Negative predictive value $=\frac{\text { true negative }}{\text { true negative }+ \text { false negative }} \times 100$ \\
\hline
\end{tabular}

group versus $0.886 \pm 0.078$ in the normal group) (Table 1).

The cutoff point of 0.8 showed the best sensitivity with excellent specificity. The condylar cutoff sign for the diagnosis of complete discoid lateral meniscus revealed greater than $76 \%$ sensitivity, $96 \%$ specificity, $95 \%$ positive predictive value, and $80 \%$ negative predictive value, in every set of measurements calculated (Table 2). None of the $50(0 \%)$ patients in the normal group had a prominence ratio less than 0.8 . In the discoid group, 38 of $50(76 \%)$ had a prominence ratio less than 0.8 . All patients with a prominence ratio less than 0.8 had complete discoid meniscus. When we set the cutoff point at $0.7,0.6$, or 0.5 , the specificity remained $100 \%$, whereas the sensitivity changed to $50 \%, 14 \%$, and $2 \%$, respectively. The positive predictive value also remained at $100 \%$ with the negative predictive values $66.7 \%, 53.8 \%$, and $50.5 \%$, respectively, according to cutoff points at $0.7,0.6$, and 0.5 .

The interclass correlation coefficients ranged from 0.88 to 0.99 , and the intraclass correlation coefficients ranged from 0.86 to 0.99 .

\section{Discussion}

The plain radiograph generally is considered a secondary tool to diagnose a lateral discoid meniscus, with MRI or arthroscopy being the primary means. However, cost and risk (with arthroscopy) make these latter two less desirable. We describe the condylar cutoff sign, a radiographic sign in knees with a discoid lateral meniscus, for diagnosis of complete discoid meniscus. We then (1) compared the prominence ratio between patients with lateral discoid meniscus and normal subjects, (2) determined the 
sensitivity, specificity, and positive and negative predictive values, and (3) determined the interobserver and intraobserver reliability of radiographic assessment of the sign.

One of the limitations of this study is we excluded patients younger than 18 years, so the applicability of the condylar cutoff sign might not apply to this group. We cannot determine from this study whether the condylar cutoff sign will be useful for diagnosis of incomplete discoid meniscus. Additionally, the difficulty in diagnosing pediatric and adolescent knee problems has been described $[11,13]$. Kocher et al. [11] suggested selective MRI does not provide enhanced diagnostic utility over clinical examination, particularly in children. Therefore, we believe our method may play an additive role. We speculate the condylar cutoff sign is the result of remodeling of the femoral condyle to the thick inner portion of the complete discoid meniscus, therefore, it is likely a much lower percentage of knees with incomplete discoid meniscus will have a positive condylar cutoff sign. Additional investigation is needed to clarify if this sign has value in the diagnosis of an incomplete discoid meniscus.

Nathan and Cole [14] suggested plain radiography had little value in the detection of a discoid lateral meniscus. Jeannopoulos [8] reported 21 cases of discoid lateral meniscus and described four cases of widened lateral joint space resulting from a thickened discoid lateral meniscus and two cases of hypoplasia of the lateral femoral condyle seen on anteroposterior radiographs. These authors stated not all cases of lateral discoid meniscus show typical radiographic findings. There have been other radiographic findings of discoid meniscus such as cupping of the lateral aspect of the tibial plateau [3] and a blunted lateral tibial spine $[1,14]$. All of these findings are considered indirect radiographic signs of discoid meniscus, and none was described with these relatively high sensitivity, specificity, and positive and negative predictive values for radiographic diagnosis of the discoid meniscus. Kim et al. [10] analyzed morphometric features of discoid and normal knees with various indirect signs on knee radiographs. Only two signs, widening of the lateral femorotibial joint space and height of the fibular head, were different between the two groups. However, as diagnostic signs of discoid meniscus, they reported sensitivity of $82 \%$ and $74 \%$, respectively, and specificity of $50 \%$ and $61 \%$, respectively, for each sign, which are too low to be clinically useful for screening or diagnosis of complete discoid meniscus.

The specificity of $100 \%$ indicates all knees in the normal group were identified as having a negative cutoff sign, meaning there were no false-positive results. This is clinically relevant in cases of complete discoid meniscus, in which a change in placement of the portal and more demanding surgical technique often are required. Higher than normal placement of the anterolateral portal often enhances the view and eases passage of the arthroscopic instrument that otherwise can be jeopardized by the thick substance of the discoid meniscus [10]. Therefore, prediction of the complete discoid meniscus by the condylar cutoff sign is valuable in diagnosis, preoperative planning, and proper portal placement. We believe the condylar cutoff sign is a simple and reliable radiographic sign for diagnosis of complete discoid meniscus in patients who have difficulty obtaining and paying for healthcare [15]. Magnetic resonance imaging also can be used to assess for the Wrisberg variant and tearing of the discoid meniscus, and is useful for preoperative planning and evaluating prognosis. If the patient has a prominence ratio less than 0.8 and has symptoms, he or she may have a high probability of having a discoid meniscus; therefore, MRI can be performed to assess for surgical treatment.

The condylar cutoff sign, as seen on tunnel-view radiographs of the knee, is useful to help diagnose a complete discoid lateral meniscus in skeletally mature patients.

Acknowledgments We thank Jong Il Sun and Hyung Kwon Ahn for radiographic measurements.

\section{References}

1. Bellier G, Dupont JY, Larrain M, Caudron C, Carlioz H. Lateral discoid menisci in children. Arthroscopy. 1989;5:52-56.

2. Davidson D, Letts M, Glasgow R. Discoid meniscus in children: treatment and outcome. Can J Surg. 2003;46:350-358.

3. Dickhaut SC, DeLee JC. The discoid lateral-meniscus syndrome. J Bone Joint Surg Am. 1982;64:1068-1073.

4. Eren OT. The accuracy of joint line tenderness by physical examination in the diagnosis of meniscal tears. Arthroscopy. 2003;19:850-854.

5. Fujikawa K, Iseki F, Mikura Y. Partial resection of the discoid meniscus in the child's knee. J Bone Joint Surg Br. 1981;63:391395.

6. Hing C, Raleigh E, Bailey M, Shah N, Marshall T, Donell S, Glasgow M. A prospective study of the diagnostic potential of the knee tunnel view radiograph in assessing anterior knee pain. Knee. 2007;14:29-33.

7. Ikeuchi H. Arthroscopic treatment of the discoid lateral meniscus: technique and long-term results. Clin Orthop Relat Res. 1982;167:19-28.

8. Jeannopoulos C. Observation on discoid menisci. J Bone Joint Surg Am. 1950;32:649-652.

9. Kaplan EB. Discoid lateral meniscus of the knee joint; nature, mechanism, and operative treatment. J Bone Joint Surg Am. 1957;39:77-87.

10. Kim SJ, Moon SH, Shin SJ. Radiographic knee dimensions in discoid lateral meniscus: comparison with normal control. Arthroscopy. 2000;16:511-516.

11. Kocher MS, DiCanzio J, Zurakowski D, Micheli LJ. Diagnostic performance of clinical examination and selective magnetic resonance imaging in the evaluation of intraarticular knee disorders in children and adolescents. Am J Sports Med. 2001;29: 292-296. 
12. Kocher MS, Klingele K, Rassman SO. Meniscal disorders: normal, discoid, and cysts. Orthop Clin North Am. 2003;34: 329-340.

13. Luhmann SJ, Schootman M, Gordon JE, Wright RW. Magnetic resonance imaging of the knee in children and adolescents: its role in clinical decision-making. $J$ Bone Joint Surg Am. 2005;87:497-502.

14. Nathan PA, Cole SC. Discoid meniscus: a clinical and pathologic study. Clin Orthop Relat Res. 1969;64:107-113.
15. Rao PS, Rao SK, Paul R. Clinical, radiologic, and arthroscopic assessment of discoid lateral meniscus. Arthroscopy. 2001;17: 275-277.

16. Resnick D, Goergen TG, Kaye JJ, Ghelman B, Woody PR. Discoid medial meniscus. Radiology. 1976;121(3 pt. 1):575-576.

17. Silverman JM, Mink JH, Deutsch AL. Discoid menisci of the knee: MR imaging appearance. Radiology. 1989;173:351-354.

18. Woods GW, Whelan JM. Discoid meniscus. Clin Sports Med. 1990;9:695-706. 